by a strong featureless continuum, whereas the already evolved site $\mathrm{E}$ shows little continuum, but strong $\mathrm{H}_{2} \mathrm{~S}(1)$ line emission.

A strong emission of $\mathrm{CH}_{4}$ was detected in the $3.50-3.56 \mu \mathrm{m}$ range 13 minutes after impact $\mathrm{H}$ on $18 \mathrm{July}, 19: 46 \mathrm{UT}$, corresponding to 5 multiplets of the $\mathrm{CH}_{4} \nu_{3} \mathrm{P}$ band centered at $3.3 \mu \mathrm{m}$ (P16 to P20). In the first image, the peak intensity at the center of the emission corresponds to a rotational temperature of about $700 \mathrm{~K}$. The temporal evolution of this emission was monitored for 30 minutes while, over the first 15 minutes, its intensity decreased exponentially with a mean timescale of 3 minutes. Emissions of $\mathrm{H}_{3}^{+}(3.53 \mu \mathrm{m})$ were also detected over most of the impact sites several hours after the impacts. The evolution of these emissions could be followed until the end of the observational run.

After the impacts, between July 22 and 31,1994 , the evolution of the Jovian atmosphere was monitored in the $2.1 \mu \mathrm{m}$ and $3.5 \mu \mathrm{m}$ regions. From July 23 to 25 , the spectra recorded at $3.5 \mu \mathrm{m}$ show $\mathrm{H}_{3}^{+}$emissions in the northern hemisphere at $+44^{\circ}$ latitude that are clearly correlated to the impact sites. The emissions are not uniformly distributed, but are concentrated at the longitudes of the impact areas. They could result from the transfer of charged particles along the Jovian magnetic field lines. At $2.1 \mu \mathrm{m}$ clear evidence for enhanced emissions at the longitudes of the impact sites at $+44^{\circ}$ latitude was found only on July 23 . For a more detailed study Jupiter was mapped by scanning the planet along its rotational axis from pole to pole. Maps of $\mathrm{H}_{2}$ and $\mathrm{H}_{3}^{+}$ were constructed allowing the analysis of the spatial and temporal evolution of these species. The spatial distribution of the emissions shows a clear trend: the later after the impacts the more emission was present in the northern hemisphere, in particular in the northern polar region. The extremely strong emissions in the northern aurora might have led to a contamination of the spectra centered at $+44^{\circ}$ latitude. Thus, although there are still indications for the presence of northern counterparts at the longitude of the impacts sites at the end of the observational run, it seems that some of the emission is actually associated to enhanced auroral activity which extends to unusually low latitudes.

\title{
NEAR-INFRARED OBSERVATIONS OF THE ENCOUNTER OF COMET SHOEMAKER-LEVY 9 WITH JUPITER WITH THE ESO-GERMAN 2.2m TELESCOPE ON LA SILLA, CHILE
}

\author{
K. Jockers (MPI Aeronomy, Katlenburg-Lindau, Germany)
}


The impact of comet Shoemaker-Levy 9 with Jupiter was observed with the IRAC-2B near-infrared camera of the European Southern Observatory (ESO) at the ESO-German 2.2m-telescope on La Silla, Chile from July 16 - 24. The IRAC-2B camera employs a NICMOS array of $256 \times 256$ pixels and in the used configuration provides a scale of $0.27 \mathrm{arcsec} /$ pixel. Data were obtained in the nights July $16 / 1723.8-3.5$ UT, July $17 / 1823.3-3.6$ UT, and July 1822.9 - 23.9 UT under rather poor meteorological conditions. Good data, collected during photometric or nearly photometric conditions, exist only for the nights July $22 / 2323.1-3.5 \mathrm{UT}$ and July $23 / 2423.1-3.5 \mathrm{UT}$, i. e. shortly after the comet impacts. Only the $\mathrm{B}$ and $\mathrm{F}$ impacts fall into the range of available observations, but no effects from these impacts are apparent in the data from the $2.2 \mathrm{~m}$-telescope.

Two kinds of data were taken, filter imaging and imaging through a tunable Fabry-Perot interferometer.

Most filter images were taken through filters centered at $2.105 \mu \mathrm{m}$ and $2.365 \mu \mathrm{m}$ with full width at half maximum 0.037 and $0.088 \mu \mathrm{m}$, respectively. At the shorter wavelength Jupiter's atmosphere is still somewhat transparent. This filter also includes the $\mathrm{H}_{2}$ quadrupole emission at $2.121 \mu \mathrm{m}$ and emissions of $\mathrm{H}_{3}^{+}$around $2.093 \mu \mathrm{m}$. The images show some of the higher atmospheric clouds of Jupiter, including the great red spot, the polar haze, and presumably some auroral $\mathrm{H}_{3}^{+}$emission. At the longer wavelength methane absorption is much stronger and Jupiter's disk appears essentially dark apart from the polar caps where some emission comes from the polar haze. Some images were taken through $\mathrm{K}$ and $\mathrm{K}$ ' wide-band filters (the $\mathrm{K}^{\prime}$ filter is like the $\mathrm{K}$ filter but has its long-wavelength cutoff already at a wavelength of $2.31 \mu \mathrm{m}$ ). The images show the development of debris clouds created after the impacts. These clouds appear equally well in both narrow-band filters which indicates their location high in Jupiter's stratosphere. The images allow to study the aging of these clouds, and the shear introduced by stratospheric winds. As some clouds were observed on their way around Jupiter's limb it may be possible to determine their geometrical height above the limb.

The tunable Fabry-Perot filter is arranged in telecentric mode, i. e. is located close to the F/33 Cassegrain focus of the telescope, outside of the cooled area of the instrument. Cold optics reimage the Cassegrain focus and the Fabry-Perot interferometer on the detector. The resolution is about 1000 . The $\mathrm{H}_{2}$ quadrupole line at $2.121 \mu \mathrm{m}$, an $\mathrm{H}_{3}^{+}$line of the hot band at $2.093 \mu \mathrm{m}$, and the continuum at $2.108 \mu \mathrm{m}$ were observed. In order to determine the flat response of the Fabry-Perot interferometer, images of a faint night glow line at $2.124 \mu \mathrm{m}$ were also taken. The proper tuning of the instrument was verified with a neon lamp. For continuum calibration Saturn's rings were observed at 
all wavelengths. Fabry-Perot interferograms were taken in all nights, and had priority in the two clear nights. A preliminary evaluation of the data shows only the gradient in the continuum radiation and no evidence for line emissions. The $\mathrm{H}_{2}$ quadrupole line was detected on July 18, 2:34 UT with ESO's NTT telescope and infrared spectrograph, i. e. with significantly larger telescope aperture and somewhat larger spectral resolution, by R. Schulz, Th. Encrenaz, J. Stüwe, and G. Wiedemann. The (stronger) auroral line at $3.533 \mu \mathrm{m}$ was also detected by this group. A more elaborate effort will be necessary to either prove detection of the lines with the Fabry-Perot interferometry or to provide upper limits on their fluxes. If the lines are detected the data will allow to study the time variability of the emissions related to the aging of the impact clouds.

\section{IMPACT L OBSERVED AT A WAVELENGTH OF $892 \mathrm{~nm}$ WITH THE SOLAR VACUUM TELESCOPE ON TENERIFE}

H. Schleicher, H. Balthasar, M. Knölker, W. Schmidt (Kiepenheuer Institut, Freiburg, Germany), K. Jockers (MPI Aeronomie, Katlenburg-Lindau, Germany

During the impact of fragment $L$ filtergrams of high spatial and temporal resolution were obtained in the methane band centered at $892 \mathrm{~nm}$ with the solar vacuum tower telescope (VTT) located at Izaña, Tenerife. The VTT has an aperture of $0.70 \mathrm{~m}$ and a focal length of $45 \mathrm{~m}$. Images were taken every $20 \mathrm{~s}$ in the prime focus with a CCD camera operated in $512 \times 512$ pixel mode. One pixel corresponds to 0.175 arcsec. The spatial resolution varied between 0.5 and 1.5 arcsec due to seeing effects. The exposure time was $2.5 \mathrm{~s}$. At 22:20:52 UT, four minutes after the impact time based on Galileo images, a first brightening above the dark southeast limb of Jupiter was recognized. A first maximum was reached after five minutes. The subsequent decline of brightness was interrupted at 22:31:52 by a sudden bright flare with a lifetime of $90 \mathrm{~s}$. Within our resolution the two recorded emission features are cospatial. The flux of this flare was $(3.4 \pm 0.2) \times 10^{-4}$ of Jupiter's total flux at this wavelength. The feature became almost invisible 19 minutes after its first detection. At 22:43 UT, 26 minutes after the impact, a new brightness enhancement rotated into view which could be identified with the dark impact signature (debris cloud) observed in the visual wavelength range.

We attribute the first brightening with the arrival of the fireball into the line of sight and the last brightness enhancement with the debris left in the 